\section{Wohin mit dem Handel?}

\author{
Lange Zeit bildeten Handel und Innenstädte eine Einheit. Doch der wirtschaftli- \\ che Wandel führt dazu, daß der Einzelhandel zunehmend die Stadt verlässt. \\ Betriebliches Rentabilitätskalkül bei der Standortwahl und die Ziele der Stadt- \\ entwicklungs- und Verkehrspolitik fallen in diesem Fall häufig auseinander. Um \\ eine Trendwende zu erreichen, müssen die Ursachen der Entwicklung analysiert \\ und neve Strategien geeignet darauf abgestimmt werden.
}

$I$

Von Christian Schneider m historischen Rückblick war es der Handel, der Städte strukturiert, den Mittelpunkt definiert und ihnen eine Identität gegeben hat. Damals wie heute ist die Stadt ein Marktplatz und die Innenstadt dessen Zentrum, das sowohl als Dreh- und Angelpunkt des öffentlichen Lebens als auch als wirtschaftliches Herz der Stadt fungiert (1). Doch heute stellt sich die Frage, ob diese historische Definition von Städten und deren Zentren noch zutrifft, da sich für den Handel die Wertigkeit von Standorten wandelt und mit ihr die existierende Symbiose „Handel und (Innen)stadt".

Bundesweit spielen sich seit Jahren räumlichstrukturelle Veränderungen im Einzelhandelssektor ab. Zunehmende Unternehmenskonzentrationen, neue Vertriebsformen und -wege sowie Maßstabsvergrößerungen der Handelseinrichtungen führten und führen unter anderem $\mathrm{zu}$ wachsenden Flächenansprüchen (2). Die daraus resultierenden Standortpräferenzen koppeln sich in zunehmendem Maße von städtischen Zentren $a b$, entwickeln ein sekundäres Versorgungsnetz, bilden eigene Zentralitäten aus und bedürfen in geringerem Maße des Städtischen.

\section{Standortwahl im Einzelhandel}

Der Standort des Einzelhandels liegt im Spannungsfeld zwischen den Zielen von Raumordnung und Landesplanung, kommunalen Interessen auf der einen Seite sowie den unternehmerischen Vorstellungen der Investoren bzw. Betreiber der Handelseinrichtung auf der anderen Seite. Gemeinden begehen bei der Standortentscheidung für den Einzelhandel ,eine Gratwanderung zwischen planerischem Dirigismus und marktwirtschaftlicher Entfaltungsmöglichkeit des Einzelhandels im Interesse der gesamten Stadtentwicklung“ (3). Im Spannungsfeld betrieblicher Gewinnmaximierung und staatlicher Maximie- rung des Allgemeinwohls sind beide Zielvorstellungen gleichermaßen nachvollziehbar. Die Versachlichung der öffentlichen Diskussion und zielgerichtete Problemlösungen bei konfligierenden Vorstellungen über die Standortwahl erfordern Kenntnisse über Motivation und Ansprüche der jeweils anderen Interessenlage.

\section{Betriebliche Perspektive}

Die Standortanforderungen für ein Einzelhandelsvorhaben variieren in Abhängigkeit von Angebots- und Vertriebsform. Es gilt jedoch immer das ökonomische Grundprinzip, dass der Standort dann wirtschaftlich tragfähig ist, wenn der $\mathrm{zu}$ erzielende Umsatz und die Kosten in einem rentablen Verhältnis zueinander stehen.

- Die Einordnung eines Standortes erfolgt zunächst unter Kostenaspekten, z.B. dem Grundstïckspreis, steuerlichen Gesichtspunkten und nach dem Zeithorizont der Vorhabenplanung und -realisierung. In die Gesamtbewertung fließen aber auch Kriterien wie die qualitative und kostenmäßige Betrachtung von Flächenzuschnitt, Stellplatzangebot und Verkehrsanbindung ein.

- Für die Abschätzung des zu erzielenden Umsatzes werden die Konkurrenzsituation und das Kundenpotential im Einzugsbereich betrachtet, um eine möglichst hohe Frequentierung zu erreichen.

Im Einzelfall können noch weitere, sogenannte „weiche Standortfaktoren“ sowie Agglomerationsvorteile Einfluss auf die Standortwahl nehmen. Insbesondere der Nähe zu anderen Handelseinrichtungen kommt dabei oftmals besondere Relevanz $\mathrm{zu}$.

Unter Verkehrsgesichtspunkten ist die Maxime für die investoren- und betriebsseitige Prüfung der Standortplanung für Einzelhandelsvorhaben klar: Es gilt, unter bestmöglichen verkehrlichen Rahmenbedingungen die meisten Kunden zu errei- chen. Bei der Gewichtung der Kriterien zur Standortentscheidung ist insbesondere die Lage des Standortes im regionalen und stadträumlichen Kontext sowie die Konkurrenz, gegen die es sich $\mathrm{zu}$ behaupten gilt und deren Marktverdrängung in „Kauf" genommen wird, entscheidend.

\section{Stadtplanerische Perspektive}

Ziel der landesplanerischen und städtebaulichen Steuerung insbesondere des großflächigen Einzelhandels ist es, zu einer geordneten Regionalund Stadtentwicklung beizutragen. Darunter ist eine wohnortnahe Versorgung periodisch nachgefragter Waren ebenso zu fassen wie Beiträge zur Verkehrsvermeidung von motorisierten Einkaufsfahrten. Wohin eine ungezügelte räumliche Handelsentwicklung führen kann, das zeigen die ostdeutschen Städte par excellence.

Nicht erst vor diesem Hintergrund wird der verträglichen Verkaufsflächenverteilung von Innenstadteinzelhandel und dezentralen Handelseinrichtungen aus stadtplanerischer Sicht besondere Aufmerksamkeit beigemessen. Definiert der Einzelhandel doch über seine zentrenund stadtbildende Kraft Zentralität, indem er über sein Attraktivitätspotenzial einen Bedeutungsüberschuss gegenüber dem umgebenden Raum generiert. Dadurch löst er Nachfrageströme bzw. Kundenfrequenzen aus. Gerade diese hohen Besucherfrequenzen sind auch die ökonomische Basis anderer städtischer Funktionen wie Dienstleistung, Gastronomie, Kultur oder Freizeit. Der Einzelhandel wirkt folglich als zentraler Impetus der Stadtentwicklung und beeinflusst wiederum die Wertigkeit des Raumes bezüglich Attraktivität, Nachfrage und damit auch der Bodenpreise.

Der Standortwahl einer Einzelhandelseinrichtung kommt auch eine besondere Bedeutung hinsichtlich der verkehrlichen Implikationen $\mathrm{zu}$. Aus dem Attraktionspotential der Einzelhandels(groß)einrichtung, also aus Größe, Warensortiment, Lage, Qualität von Erreichbarkeit und Erschließung sowie der Anzahl an Stellplätzen, leitet sich das Verkehrsaufkommen und die Verkehrsmittelwahl im Einkaufsverkehr ab (4). Mit zunehmender Verkaufsflächendimensionierung erweitert sich der Einzugsbereich, steigt die Autokundenorientierung und damit auch die Verkehrserzeugung.

Zentrale und verbrauchernahe, sogenannte integrierte Standorte tragen zur Auslastung des öffentlichen Verkehrs bei. Fällt die Wahl auf einen städtebaulich nicht integrierten Standort, 
muss die Stadt hingegen oftmals zusätzlich in die Verkehrserschließung investieren. Teilweise weisen nicht integrierte Standorte ein Autoverkehrsaufkommen von über 80 Prozent, häufig sogar über 95 Prozent auf. Dies gilt selbst dann, wenn attraktive Anbindungen an öffentliche Verkehrsangebote bestehen (5).

Die räumliche Standortsteuerung und Dimensionierung von Einzelhandels(groß)einrichtungen ist daher eine zentrale Komponente der Stadtentwicklung.

\section{> Zielkonflikte}

In letzter Zeit stehen sich betriebliche sowie stadtund verkehrsplanerische Ziele zunehmend diametral gegenüber. Für Investoren und Betreiber erweist sich die Realisierung von Einzelhandels(groß)vorhaben an städtebaulich nicht integrierten Standorten sowohl nach Kosten-, Rentabilitäts- als auch Wettbewerbskriterien häufig als vorteilhafter. Der Handlungsdruck wird durch einen steigenden Verdrängungswettbewerb und sinkende Margen verstärkt. Die zunehmende Flexibilisierung, Pluralisierung und Automobilisierung des Kundenverhaltens verschärfen die branchenspezifische wie auch standortbezogene Konkurrenzsituation zwischen integrierbaren und nicht integrierten Handelsstandorten. Dieser Trend hin zum Handel auf der grünen Wiese stellt sich in der stadtökonomischen Gesamtbilanzierung für die Gemeinde häufig nachteilig dar. Nicht integrierte Standorte erfordern oftmals zusätzliche Erschließungs- und Infrastrukturkosten, induzieren zusätzliches Autoverkehrsaufkommen, können den Freiflächenverbrauch steigern sowie zu unverträglichen Kaufkraftumverteilungen und daraus folgend zu negativen städtebaulichen Auswirkungen in den Zentren beitragen, wodurch wiederum private wie öffentliche Investitionen in den Zentren entwertet werden können.

Wenn jedoch die Flächenansprüche von Einzelhandels(groß)einrichtungen weiter steigen, was aktuelle Entwicklungen wie Einkaufszentren, Fachmarktzentren, Fachmärkte oder Urban-Entertainment-Center und Factory-Outlet-Center belegen, so wird deren (Nicht-) Integrierbarkeit zukünftig zur entscheidenden stadtentwicklungsrelevanten Fragestellung avancieren. Die zusätzliche Induzierung von motorisiertem Individualverkehr, negative Auswirkungen und Belastungen inbegriffen, gerät unberechtigterweise in der Planungspraxis häufig in den Hintergrund der ,öffentlichen“ Diskussion.

\section{Konsequenzen für die Standortentwicklung}

Es spricht also vieles, insbesondere stadtökonomische Gesichtspunkte, für integrierte Standorte von Einzelhandels(groß)betrieben, auch wenn sie in Innenstädten als Verkehrserzeuger fungieren und bereits vorhandene Belastungen steigern können. Durch die richtige räumliche Standortwahl kann hier aber bereits an den Ursachen und nicht an den Symptomen der Verkehrsentstehung angesetzt werden.

Um diese für die Investoren akzeptabel zu machen, müssen jedoch Lösungen für die Faktoren gefunden werden, die aus betrieblicher Sicht gegen integrierte Standorte sprechen. $\mathrm{Zu}$ nennen sind hier die in vielen Städten vorhandenen Flächenengpässe, Kosten für Gebäudeumnutzungen oder Brachflächen, Stellplatzablöseverordnungen usw. Doch auch die zeitgemäße Präsentation der Waren oder die Erreichbarkeit und „Kostenlosigkeit der Stellplatzanlagen“ stellen betriebliche Standortprofile dar. Diese betrieblichen Rentabilitätsfaktoren, die von Investoren in der Projektentwicklung mitunter angeführt werden, um Gemeinden im Wettbewerb um Investitionen, Steueraufkommen und Image gegeneinander auszuspielen, lassen sich durchaus auch an integrierten Standorten realisieren: Wille, Ideenreichtum und Gesprächsbereitschaft von Politik, Verwaltung und anderen Entscheidungsträgern vorausgesetzt.

Dass Wege und Möglichkeiten der Standortwahl von Einzelhandels(groß)einrichtungen existieren, die öffentlichen Planungs- und unternehmerischen Wirtschaftlichkeitsansprüche in Einklang zu bringen, deuten aktuelle Entwicklungen an:

1.Städte und Gemeinden stellen zusehends kommunale Einzelhandelskonzepte auf, die die weitere räumliche Einzelhandelsentwicklung fixieren und fördern sowie dem Handel und Investoren Planungs- und Investitionssicherheit liefern (6).

2. Auch auf regionaler Ebene wird die Notwendigkeit gemeinsamer Entwicklungskonzepte zusehends erkannt. Interkommunale Abstimmungen zur regionalen Einzelhandelssteuerung und Orientierung an städtebaulich verträglichen und absatzwirtschaftlich tragfähigen Spielräumen der Handelsentwicklung finden derzeit Eingang in die Praxis. Entsprechende Verbindlichkeiten und akteursspezifische Zuständigkeiten sind dabei durchaus variabel und optional gestaltbar.
3. In Zeiten einer steigenden Bedeutung von Kooperationsformen öffentlicher Planung und privater Projektentwicklung liefert darüber hinaus der Abbau von Informationsdefiziten bezïglich der jeweiligen Anforderungen der anderen Akteursseite Möglichkeiten zur frühzeitigen Versachlichung der Entscheidungsfindung.

Die Nutzung derartiger und weiterer Entwicklungsoptionen mag für die Städte wie für die Projektentwickler mitunter eines größeren Aufwandes bedürfen. Beide Seiten können aber im Sinne einer stadt- und regionalverträglichen Standortentwicklung des Einzelhandels Beiträge zur Ausbalancierung seiner zentralisierenden und verkehrserzeugenden Wirkungen leisten.

\section{Anmerkungen}

(1) Junker, Rolf/ Stefan Kruse: Perspektiven des Handels und deren Bedeutung für die Entwicklung von Zentren. In: Informationen zur Roumentwicklung, Heft 2/3, 1998, S. 133-139.

(2) Hatzfeld, Ulrich/ Wolfgang Roters: Zentrum - Peripherie: Was sollen wir wollen oder: Spielen auf Zeit? In: Informationen zur Raumentwicklung, Heft 7/8, 1998, S. $521-535$.

(3) Knop, Bernd: Standortwahl im Einzelhandel. Staatliche Steuerung in der Marktwirtschaft? - Zur Legitimation aus ökonomischer Sicht - In: RoumPlanung Nr. 55, 1991, S. 213-216, hier S. 215 .

(4) Kleinstädte verzeichnen zum Beispiel im Einkaufsverkehr größere Pkw-Anteile als GroBstädte. Vgl. Bundesarbeitsgemeinschaft der Mittel- und Großbetriebe des Einzelhandels; Einkaufsverkehr - Ergebnisse der Kundenbefrogung. Köln 1993 und 1997.

(5) Arbeitsausschuß "Grundsatzfragen der Verkehrsplanung" der Forschungsgesellschaft für Straßen- und Verkehrswesen (FGSV): Verkehrliche Wirkungen von Großeinrichtungen des Handels und der Freizeit, FGSV . Arbeitspapier Nr. 49, Köln 1999.

(6) Kruse, Stefan/Christian Schneider: Von der Reaktion zur Aktion - Einzelhandelssteuerung und -förderung durch Einzelhandelskonzepte. In: Standort - Zeitschrift für angewandte Geographie, Heft 2/1998, S. 27-30.

\section{Der Autor}

Christian Schneider ist wissenschaftlicher Mitarbeiter bei Junker und Kruse Stadtforschung-Stadiplanung. Kontakt: Markt 5, 44137 Dortmund, Tel. 0231/ 557858-0, Fax - 50,

E-mail: schneider@junker-kruse.do.uunet.de 
(c) 20I0 Authors; licensee IÖW and oekom verlag. This is an article distributed under the terms of the Creative Commons Attribution Non-Commercial No Derivates License (http://creativecommons.org/licenses/by-nc-nd/3.o/), which permits unrestricted use, distribution, and reproduction in any medium, provided the original work is properly cited. 